estimate the viscous flow pressure, which is equal to the difference between the two pressures (line $c$ ). So the actual intravascular pressure recorded by the catheter tip transducer is the sum of viscous flow pressure and gravitational pressure (see figure; line $c+(-$ line $b)=$ line $a)$. Thus, the drop in measured pressure down the jugular vein can be explained on the basis that the fall in viscous flow pressure is greater than the increase in gravitational pressure down the jugular vein of the standing giraffe.

Pedley estimated' from the data of Hargens et al. a mean pressure gradient of about $80 \mathrm{~mm} \mathrm{Hg}$ per m length of jugular vein coming from viscous resistance to flow (in agreement with line $c$ ). $\mathrm{He}$ applied this value to the Poiseuille equation to calculate the flow rate through the jugular vein, assuming the diameter of 2.5 $\mathrm{cm}$ reported by Goetz et al. ${ }^{3}$. Although Goetz et al. reported the external diameter of the vessel, which appeared collapsed, the flow rate calculated by Pedley is impossibly high. He argued on this basis that the jugular vein in the standing giraffe must be severely col- lapsed which, he believes, is inconsistent with the siphon mechanism.

I disagree with this view because no matter how collapsed the jugular vein is, the siphon mechanism should operate if there is a continuous column of liquid. It is inconceivable that the blood column in the jugular vein of the upright giraffe is broken into separate, disconnected compartments.

To support this view, I performed an experiment in which I attached a $90-\mathrm{cm}$ length of surgical drain tubing (Penrose) with a diameter of $1 \mathrm{~cm}$ to a thick $5 / 16$ inch-bore latex tubing. I filled these with water and a siphon established from a water tank. The lower end of the highly collapsible drain tube was about $107 \mathrm{~cm}$ below the level of water in the tank. During flow, amounting to about 1,800 millilitres per minute, the drain tube is practically flat, showing that a severe collapse of the thin tube does not abolish the siphon mechanism. The flow occurs essentially from the corners of the flat tube which, on cross section, looks somewhat like a dumbbell as demonstrated by Katz et al.$^{4}$. This model demonstrates unequivocally

\section{Highly conserved repeats in heat-shock introns}

SiR-The heat-shock cognate proteins of $M_{\text {r }}$ (relative molecular mass) 70,000 (Hsc70) are very similar in sequence to the stress-inducible heat-shock proteins of $M$ 70,000 (HSP70) but are constitutively expressed $^{1.2}$. The recently reported sequences of the rat ${ }^{3}$ and human ${ }^{4} \mathrm{Hsc} 70$ genes reveal that each contains eight introns. During computer-assisted analysis ${ }^{5}$ of the sequences, we found a highly conserved sequence that occurs once in introns 5,6 and 8 of both the rat and human sequences ( $a$ in the figure). Each of these regions is approximately 82 per cent identical to the other five over a span of about 90 nucleotides, and each is flanked by short inverted repeats. We find no other similarity between the rat and human intron sequences or between any of these introns and any of the sequences currently available in the GenBank database.

There is also a second type of repeat ( $b$ in the figure), which occurs only in introns

1 and 3 of the human Hsc70 sequence. These repeats are 84 per cent identical over a stretch of 156 nucleotides.

If the repeats that occur three times each in the rat and human introns have been in place since before the divergence of these two species, then the extent of sequence similarity suggests that they have a functional role. We can find no similar published phenomenon.

\section{Howard Hughes Medical Institute,}

Dallas,

Texas 75235, USA

Department of Microbiology,

Gabriele I. Mues

University of Texas Health Science Center. Dallas,

Texas $75235, U S A$

1. Craig, E.A. CRC Crit. Rev. Biochem. 8, 239 (1986)

2. Pelham, H.R.B. Cell 46, 959 (1986)

3. Sorger, P.K. \& Pelham, H.R.B. EMBO J. 6, 993 (1987)

4. Dwornzak, B. \& Mirault, M.-E. Nucleic Acids Res. 15,518 (1987).

. 6. Weiher, H. et al. Science 219,626 (1983).

\begin{tabular}{|c|c|}
\hline \multicolumn{2}{|l|}{$a$} \\
\hline CONSENSUS & TCGCTGTGATGATTGATTCCAAAACCATTCGTAGTTTCCACCAGAAA-T-G-CTGATGTTGGCTAGTTCCTTCCTTGGATGTCTGAGCGA \\
\hline Rat I5 (2671) & 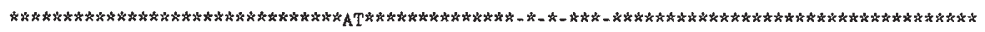 \\
\hline Human I5 (2942) & 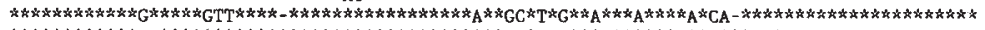 \\
\hline Rat I6 (3084) & 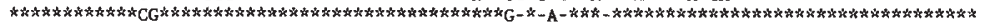 \\
\hline Human I6 (3469) & 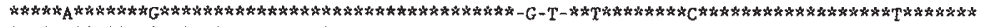 \\
\hline Rat I8 (3822) & 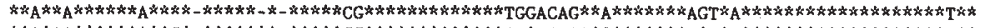 \\
\hline Human $18(4202)$ & 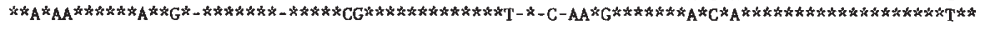 \\
\hline \multicolumn{2}{|l|}{$b$} \\
\hline $\begin{array}{l}\text { Human Il }(676) \\
\text { Human I3 }(1784)\end{array}$ & 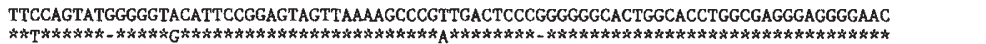 \\
\hline & 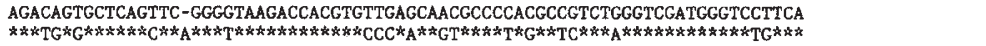 \\
\hline \multicolumn{2}{|c|}{$\begin{array}{l}a \text {, Alignment of the similar regions found in rat and human Hsc } 70 \text { introns } 5,6 \text { and } 8 \text { to a } \\
\text { consensus sequence derived from the alignment. } b \text {, Alignment of similar regions in introns } 1 \\
\text { and } 3 \text { in the human Hsc } 70 \text { gene. Identity is indicated by asterisks. Numbering as in refs } 3 \text { and } 4 \text {. }\end{array}$} \\
\hline
\end{tabular}

the operation of the siphon mechanism in severely collapsed tubes.

In view of the uncertainties about the functional configuration of the giraffe's jugular vein, it seems that further studies are necessary. The following may be suggested: (1) injection of radiopaque material into the upper jugular and visualization of the vessel; (2) study of the blood flow down the jugular with ultrasound; and (3) measurement of the viscous flow pressure not directly at various levels of the jugular with a fluidfilled catheter inserted at the lower end of the vein and pushed up, the transducer being located at the level of the right atrium. This method will exclude the gravitational component of pressure. Such studies will shed further light on the haemodynamics of venous return from the head of the giraffe and provide data about the configuration of the vein in vivo and the validity of the siphon mechanism.

HENRY S. BadeEr

Department of Physiology,

Creighton University School of Medicine, Omaha,

Nebraska 68178, USA

1. Hargens, A.R., Millard, R.W., Pettersson, K. \& Johansen, K. Nature 329, 59-60)(1987)

Pedley, T.J Nature 329,13-14 (1987)

3. Goetz, R.H et al. Circ. Res. 8, $1049-1058$ (1960)

4. Katz, A.1., Chen, Y. \& Moreno. A.H. Biophys. J. 9, 1261 $1279(1969)$.

\section{Can the new polio vaccines take the heat?}

SIR-The replacement of amino-acid sequences from the antigenic site on poliovirus type 1 by analogous antigenic sites from either type 2 or type 3 has produced cultivable chimaeras capable of stimulating production of antibodies against both serotypes used in the construction of the chimaera, but, it is hoped, without the rare cases of poliomyelitis normally associated with the use of whole, attenuated type 2 and 3 strains as vaccines ${ }^{1.2}$.

Such chimaeric poliovirus vaccines could eliminate vaccine-associated poliomyelitus in developed countries, but are unlikely to have any effect in reducing the $200,000-2,000,000$ cases of paralytic poliomyelitis that occur annually in developing countries'. The record of presently available poliovirus vaccines in developing countries is dismal, as they are destroyed by temperatures of $35-45^{\circ} \mathrm{C}$, as well as by exposure to extremes of sunlight, drought and humidity ${ }^{3}$. Exposure of potential vaccine strains to such extremes of environment in the laboratory might be of greater use in selecting strains for the developing world.

\section{M-122, Greater Kailash-II,}

S.C. ARYA

\footnotetext{
Hogle, J.M. Nature 332, 13-14 (1988)

Burke, K.L., Dunn, G., Ferguson, M., Minor, P.D. \& Almond, J.W. Nature 332, 81-82 (1988)

3. Arya, S.C. Ann. Inst. Pasteur Virol. 138, 527-528 (1987)
} 